\title{
Correction to: longitudinal analysis of physical activity, sedentary behaviour and anthropometric measures from ages 6 to 11 years
}

Phillipp Schwarzfischer ${ }^{1}$, Dariusz Gruszfeld ${ }^{2}$, Piotr Socha ${ }^{3}$, Veronica Luque ${ }^{4}$, Ricardo Closa-Monasterolo 4 , Déborah Rousseaux ${ }^{5}$, Melissa Moretti $^{5}$, Benedetta Mariani ${ }^{6}$, Elvira Verduci ${ }^{6}$, Berthold Koletzko $^{1}$ and Veit Grote ${ }^{1 *}$

\section{Correction to: International Journal of Behavioral Nutrition and Physical Activity (2018) 15:126 https://doi.org/10.1186/s12966-018-0756-3}

Following publication of the original article [1], the author reported an error in the 'Conclusion' section as follows:

Incorrect statement in published article: "On the other hand, a shorter time spent in MVPA predicted a reduced BMI over a 5-year period."

Corrected statement: "On the other hand, a shorter time spent in MVPA predicted an increased BMI over a 5-year period."

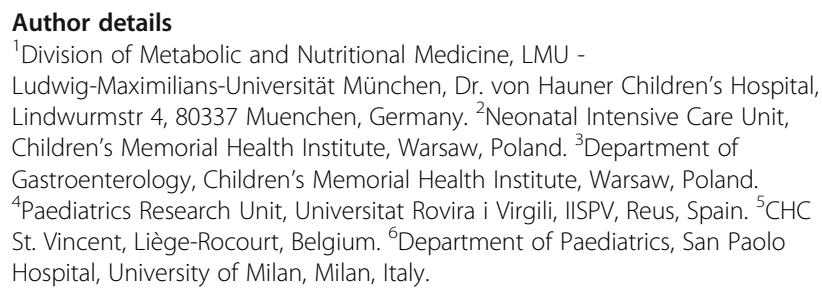

Published online: 04 March 2019

\section{Reference}

1. Schwarzfischer et al. Int J Behav Nutr Phys Act (2018) 15:126 doi https://doi. org/10.1186/s12966-018-0756-3

\footnotetext{
* Correspondence: veit.grote@med.uni-muenchen.de 'Division of Metabolic and Nutritional Medicine, LMU Ludwig-Maximilians-Universität München, Dr. von Hauner Children's Hospital, Lindwurmstr 4, 80337 Muenchen, Germany
} 